\section{FAQ's About Computer Ergonomics and Workstation Injuries}

Jonathan Bailin, Copyright: 1992-195

Stop, read, and listen to your body! Repetitive Strain Injury (RSI) is now endemic to the keyboarding population. Find out how to prevent RSI's, like Carpal Tunnel Syndrome, by reading the FAQ at the Typing Injury Web Site [http://www.cs.princeton.edu/ dwallach/tifaq/general.html] or at the Usenet Newsgroup [misc.health.injuries rsi.moderated] where you can ask questions of an expert medical panel.

Don't be a statistic! If you work more than a few minutes a day in repetitive tasks like keyboarding, "preventing ${ }^{*} \mathrm{RSI}$ is the key. Treatment outlooks can be dismal. Please tell a keyboarding friend.

\section{Q. What is "ergonomics"?}

A. Ergonomics is the science of adjusting your work environment to fit your body and make it most comfortable.

2. Q. What is the best room lighting to help reduce eye strain?

A. A mixture of fluorescent and incandescent light is usually most pleasing The most important aspect of lighting is to reduce glare and bright reflections from your screen, nearby glass, or shiny surfaces. Since light conditions change during the day this may require several adjustments while working. If you smoke while keyboarding, be sure to clean your screen frequently as water vapor and smoke make a potent film forming process.

3. Q. What is the best position for the monitor at my workstation?

A. Many make the common mistake of putting the monitor, the keyboard, or both off to one side on a desk. If you perform more than a few minutes of keyboarding a day, the keyboard and monitor should be placed directly in front of your normal sitting position. The screen should be $18-30$ inches from your eyes or about an arm's length.

4. Q. Is there an optimum height for my monitor?

A. Yes. The top of the monitor should be at eye level because the eyes are at their most comfortable position straight ahead but slightly down ward. This is why reading lenses in bifocal glasses are placed just below the horizontal plane.

On the topic of eye correction, make sure your eyes are 20/20 and that if you do need correction your optometrist should know about the amount of your monitor use and its distance from your eyes. A correction just for monitor use may be necessary. Be sure to look away from your screen at least every 30 minutes and focus on something over 20 feet away.

\section{Q. Is there an optimum screen brightness and color scheme to help} prevent eye strain?

A. Black characters against a light gray background are often easiest on the eyes for long periods. Contrast and brightness should be adjusted to create the brightest screen without blurring.

6. Q. What other accessories and placement are important?

A. Frequently used items should be within arms reach from your key boarding position. A document holder should be at the same height and distance as the screen so that your eyes don't need to change focus frequently. Frequent telephone use should utilize a headset to avoid bending the neck while keyboarding. Remember that many RSI's begin with nerve insult in the neck and shoulders.

7. Q. What is the most healthy posture for my wrists while typing?

A. The best position is neutral. In other words, the knuckles, wrist, and top of the forearm should form a straight line.

8. Q. Can a wrist pad sitting in front of the keyboard be used during keyboarding?

A. The neutral position described in \#7 can not be achieved while in contact with most commercial wrist pads. For this reason keyboarding is best performed formed from a "floating" wrist position. Contact wristpads for rest periods only. Frequent rest becomes necessary with floating wrists because it tends to emphasize shoulder muscle contraction. Don't forget to use the lightest possible finger pressure during keying.

9. Q. What is the best elbow and shoulder position while keyboarding?

A. The elbows should form a 90 degree angle while *hanging ${ }^{*}$ at your sides from the shoulders. Rarely do chairs with armrests allow this position. It is "very* important that the shoulders remain relaxed in a lowered position during keyboarding (see \#6).

\section{Q. What is the best seat height for keyboarding?}

A. It is most important that seat height should allow the upper body postures described in $\# 7$, $\# 8$, and $\# 9$. This upper body posture is most responsible for reducing risk of injury. Once this is accomplished, the feet should be flat on the floor.

If the resulting seat height prevents the feet from resting flat on the floor, a foot rest is necessary. This should allow the lower legs to be vertical and thighs horizontal.

\section{Q. What should I look for in the backrest of a chair?}

A. Expensive motors and adjustable sections are not necessary if the backrest has firm support for the inward curve of the lower spine (lumbar) and outward curve of the upper spine (thoracic). Whether you need upper body support to help keep your torso and head vertical is a matter of preference.

12. $Q$. What other characteristics of a chair are important?

A. The seat of the chair should be large enough to accommodate frequent changes in position and firm enough to allow your weight to be supported through the buttocks not the thighs. If others will use your chair, easy height adjustment is a must.

13. Q. How often should I change positions and take breaks during keyboarding?

A. You should change your sitting position at least every 15 minutes. Active breaks should be taken at least every 30 minutes especially for those who perform more than 2 or 3 hours of keyboarding a day. Microbreaks should occur more often.

\section{Q. What is an "active break" and a "microbreak"?}

A. An active break occurs when you stop keyboarding to do other things like take phone calls, file papers, or get up to get a drink of water. An active break should also include specific exercises. These exercises should also be done during keyboarding microbreaks which occur while seated at your work station.

15. Q. What are some of the best exercises for keyboarding microbreaks while seated?

A. The "Shoulder Blade Squeeze" is performed by raising your forearms and pointing your hands to the ceiling. Push your arms back, squeezing your shoulder blades together. Hold for at least 5 seconds and repeat 3 times.

"Eye Palming" is performed by placing your elbows on your desk, cup your hands, close your eyes, and place your eyelids gently down onto your palms. Hold this position for 1 minute while breathing deeply and slowly. Then uncover your eyes slowly.

The "Arm \& Shoulder Shake" is performed by dropping your hands to your sides then shake your relaxed hands, arms, and shoulders gently for at least 5 seconds and repeat 3 times.

"Spanning" is performed by placing your arms straight in front of you and spreading your fingers as far as possible for at least 5 seconds and repeat 5 times. This exercise was made famous by planists. With the arms extended in front of you spanning can be combined with a "Forearm Stretch" by turning the hands so that their backs touch then turning them so that the palms face the ceiling.

These are only a few key exercises. Many more are useful for preventing repetitive strain. Try to find the best series for your areas of tension and particular relaxation needs. Frequent breaks yield better long term productivity!

16. Q. I've heard many names for keyboard injuries. What do they all mean? 
A. Repetitive Strain Injury (RSI) is a description of an injury associated any repetitive activity such as hammering, piano playing, truck driving, computer use, or even shaking hands. Occupational Overuse Syndrome (OOS), Cumulative Trauma Disorder (CTD), and Work Related Upper Limb Disorders (WRULD), are all equivalent expressions to RSI.

Tendonitis and tenosynovitis are characterized by inflammation of tendons or their surrounding sheaths, respectively. Both of these RSI disorders usually begin as mildly aggravating and, given bad habits, may quickly progress to be severely debilitating. These common RSI injuries also add to the difficulty of proper diagnosis and deserve greater recognition. These tendon inflammations usually occur before full blown Carpal Tunnel Syndrome.

Carpal Tunnel Syndrome (CTS) is a specific, severe, and debilitating form of RSI which describes a squeezing of the median nerve as it runs to hand. The nerve is squeezed by swollen tendons surrounding it as they cross through a tunnel made by ligaments at the inside of the wrist.

The National Center for Health Statistics estimates at least 1.89 million people have Carpal Tunnel Syndrome. Many experts feel that CTS is also associated with nerve compression symptoms in the chest or shoulders. ${ }^{* \star A} A l l$ RSI symptoms should receive immediate medical attention from physicians experienced in RSI.**

17. Q. Advice by Health Care Practitioners often includes a collection of terms from a kinesiology course. Which ones do I need to know to help identify my own workstation ergonomic problems?

A. Standing with the arms at your sides, palms facing forward, "flexion" is folding of any joint of the body so that the angle between the parts decreases in the forward direction, except at the knee and toes. Returning the joint to its straight position requires "extension".

A joint which continues its extension past its straight posture is in "hyperextension". This occurs in the hand and wrist when you pull the fingers back.

Standing with your arms at your sides, palms facing forward, "pronation" is the turning of your hand so that you thumb points toward your leg.

"Supination" is the opposite movement.

18. Q. Why does it seem like RSI from keyboarding has become such a big problem recently?

A. One reason why RSI is becoming more prevalent is because computers are now allowing us to do more office tasks which formerly allowed us to change activity. For example, a typewriter at one time required using a return carriage, "white out" for mistakes, breaks for paper installation, and getting up to file papers in a cabinet

Computer word-processing now eliminates these "microbreaks". In short, computers have greatly simplified office activity, an advance that has at least one important disadvantage. The danger is found in the possibility for long duration, continuous, and relatively motion free, precise, muscular activity called "static exertion". Humans were not well "designed" for this.

19. Q. What lifestyle changes can I make to reduce the chances of RSI?

A. Two main themes permeate ergonomic study of RSI prevention; posture and relaxation. Appropriate postures are necessary to keep the strain of performing work in a near stationary position (static exertion) to a minimum. But even the best postures can fall prey to overload when with bad habits.

Relaxation is critical to the body's resilience, its ability to recover from keyboarding. Office workload dynamics can have a great influence on the risk of RSI. Try to promote office policies which emphasize steady work load schedules and avoid, or at least distribute, crises deadlines.

Stretching and strengthening active muscles promotes relaxation. Relax ation is as important for prevention of RSI symptoms as it is for general wellbeing. Take a new, more active role in promoting your general fitness both at and away from work. If you don't exercise regularly and your over 40 , get clearance from a physician to add walking, bicycling, or swimming to your weekly schedule on three separate days.

Cut down on stimulants like coffee, sweets, or nicotine and spread healthy snacks and water intake throughout the day. Keep water at your desk as it makes for a smart microbreak. The first symptom of dehydration is fatigue, not thirst!

Fruit and vegetable snacks prevent mid-morning and mid-afternoon blood sugar drops. These dips can effect alertness, mood, productivity, and decision making. A diet emphasizing complex carbohydrates, reliable sleep ing patterns, and time for yourself can do wonders for 9 to 5 productivity, not to mention your own well-being. All habits and practices that promote relaxation are necessary to stop the threat of RSI. Good Luck.

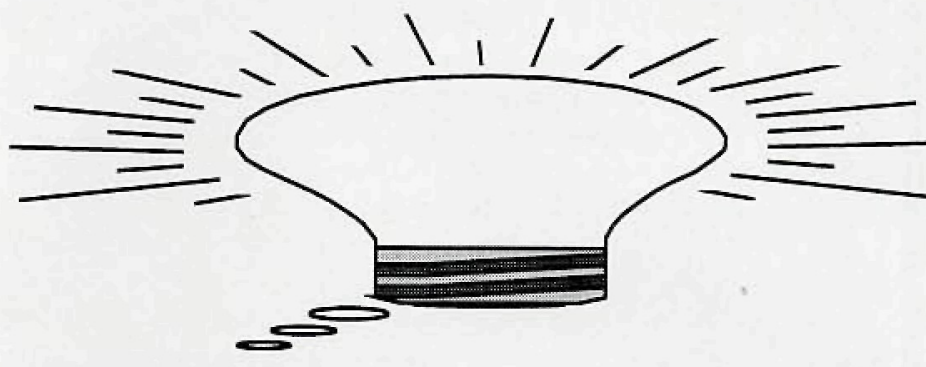

\section{Microphilosophy}

Robert $V$. Blystone Trinity University, Department of Biology

How do we know if what we see through the microscope is real? There is a group of social scientists who are known as social constructionists. Of this group there are those known as deconstructionists. And of this group there are those who are engaged in the "Science Wars". These individuals more or less advocate that what scientists do is a construction of their mind and not necessarily an expression of nature. Some continue to argue that the actions or constructions of scientists are done in part to get money to play around in the lab. Some of these people ask that federal funding be curtailed for these constructions of people called scientists. In some way the arguments remind one of animal rights activists attacking medical research.

Alan Sokal wrote an article call "Transgressing the Boundaries:..." and it was accepted and published in the journal Social Text. This journal with a circulation of about 800 is a leading journal for social constructionists and a place where debates about the science wars have been taking place. Sokal, an avowed leftist physicist, wrote "Transgressing" as a hoax and immediately proclaimed so when the Social Text article was published. His purpose was to show that the Science Wars advocaies were on thin intellectual ground. You may wish to read several recent articles of Academe (an AAUP publication) that tries to put this all into focus.

So back to the microscope. Those of us who do microscopy know that much of what we look at is a construction. The tissue is dead, chemically altered, stained, dehydrated, infiltrated, and sectioned into to little pieces... AT BEST. Clearly we are constructing what we hope is a correct interpretation of nature. Akin to walking through a graveyard and trying to guess what really happened in the living lives of the people under the tombstones. We also know in the best sense of Popper that we are self doubting and trying to better (disprove) much of what was published before. These social constructionists do not seem to understand any of this. Just like I don't understand why people watch soap operas on daytime television.

How do we tell a public what we see through the microscope is real? It is a kind of "Daddy, why is the sky blue?" question. As microscopists we have a responsibility to address the question and help the public understand what we see is "real" and represents nature. And yes, it is a construction of sorts but that is what science is all about: humans' feeble attempt to reconstruct the beauty of nature... but not in the sense of the deconstructionists.

I recognize this is an unusual observation, but believe it to be an important issue as it can dramatically affect the findings that many of us share. 